\title{
Undescended Parathyroid Adenomas: A Leading Cause of Persistent Primary Hyperparathyroidism
}

\author{
Ankita Roy ${ }^{1}$, Muhammad H Khan², Joseph Frazzetta ${ }^{3}$, Adam S Kabaker ${ }^{4}$, Steven De Jong 5
}

\begin{abstract}
Aim:We present the case of a 64-year-old man with symptomatic primary hyperparathyroidism (PHPT) who was found to have a rare undescended parathyroid adenoma (UPA). While UPAs have a low prevalence, they are challenging cases for endocrine surgeons. We include background information and a summary of the literature of UPAs and presentation, workup, management, and outcomes of this patient.

Introduction: Undescended parathyroid adenomas are rare ectopic glands that are found above the upper pole of the thyroid gland. ${ }^{1}$ They are difficult to localize on routine imaging and require strategic preoperative planning to determine the surgical approach.

Case description: A 64-year-old man presented to our tertiary care center with symptomatic PHPT. Sestamibi with SPECT/CT showed a $1.4 \mathrm{~cm}$ focus of uptake in the left upper neck that was suspicious for an ectopic UPA.

Conclusion: With appropriate preoperative imaging and planning, the patient was able to undergo curative minimally invasive parathyroidectomy. The patient had an uncomplicated postoperative recovery with resolution of symptoms and normalization of calcium and parathyroid hormone (PTH) levels.

Clinical significance: Undescended parathyroid adenomas are difficult to locate and are a leading cause of persistent PHPT, failed cervical explorations, and patient morbidity. ${ }^{2}$ Preoperative imaging is key to localizing these adenomas for successful first-time explorations and minimally invasive parathyroidectomies.

Keywords: Case report, Minimally invasive parathyroidectomy, Primary hyperparathyroidism, Undescended parathyroid adenoma.

World Journal of Endocrine Surgery (2020): 10.5005/jp-journals-10002-1306
\end{abstract}

\section{BACKGROUND}

Primary hyperparathyroidism (PHPT) affects nearly 100,000 Americans and 1 in 500 women over the age of 40 years annually. It is the most common cause of hypercalcemia in the outpatient setting and leads to bone pain, fatigue, neuropsychiatric manifestations, kidney stones, and constipation. Nearly $85 \%$ of cases are caused by a single parathyroid adenoma. In these cases of the single-gland disease, $6-16 \%$ of cases are caused by an ectopic parathyroid gland. ${ }^{3}$ Ectopic parathyroid adenomas are defined as glands that localize with tissues of the same embryological origin and are found in anomalous locations.'

The superior and inferior parathyroid glands both originate from endoderm. The third branchial (pharyngeal) pouch forms the superior parathyroid glands, which descend during development and remain close to the lateral lobes of the thyroid. The fourth branchial (pharyngeal) pouch forms the inferior parathyroid glands, which take a longer path of migration along with the thymus. ${ }^{4,5}$ Inferior parathyroid glands are thus more likely to be found in ectopic locations since they travel farther during development. ${ }^{5}$

The most common locations for ectopic parathyroid glands are within the thymus, retropharyngeal, mediastinal, intrathyroidal, within the carotid sheath, and in association with the vagus nerve. ${ }^{6}$ Ectopic superior parathyroids are most commonly found within the tracheoesophageal groove. Ectopic inferior parathyroids are most commonly found within the thymus. ${ }^{2}$ Undescended parathyroid adenomas (UPAs) are defined as being located $1 \mathrm{~cm}$ or more superior to the upper pole of the thyroid gland. ${ }^{7}$ While the exact prevalence is difficult to ascertain, it is estimated that UPAs make up $<1 \%$ of all parathyroid adenomas ${ }^{8}$ and represent $2-7 \%$ of all ectopic parathyroid adenomas. ${ }^{9}$ The following is a case presentation of a rare UPA in a patient with symptomatic PHPT.
${ }^{1}$ Department of Surgery, Loyola University Medical Center, Feinberg SOM, Northwestern Medicine, Maywood, Chicago, Illinois, USA

2,3 Department of Surgery, Loyola University Medical Center, Maywood, Illinois, USA

${ }^{4}$ Department of Surgery, Loyola University Chicago, Maywood, Illinois, USA

${ }^{5}$ Department of General Surgery, Loyola University Medical Center, Maywood, Illinois, USA

Corresponding Author: Ankita Roy, Loyola University Medical Center, Feinberg SOM, Northwestern Medicine, Maywood, Illinois, Chicago, USA, Phone: +1 8134840418, e-mail: royankita@gmail.com

How to cite this article: Roy A, Khan MH, Frazzetta J, et al. Undescended Parathyroid Adenomas: A Leading Cause of Persistent Primary Hyperparathyroidism. World J Endoc Surg 2020;12(3):132-135.

Source of support: Department of General Surgery, Loyola University Medical Center

Conflict of interest: None

\section{Case Description}

The patient is a 64-year-old man who was incidentally found to have hypercalcemia on routine lab testing 3-4 years before his presentation at Loyola University Medical Center's (LUMC's) Endocrine Surgery Clinic. The patient had reported minimal symptoms of urinary frequency, so this finding was not worked up at the time. Routine lab testing was repeated when the patient changed primary care physicians. In June of 2020, further workup was significant for a calcium level of $11.2 \mathrm{mg} / \mathrm{dL}$ (normal 8.5-10.2 $\mathrm{mg} / \mathrm{dL}$ ) with a parathyroid hormone (PTH) level of 99 (normal $10-55 \mathrm{pg} / \mathrm{mL}$ ), biochemically confirming the diagnosis

( ) Jaypee Brothers Medical Publishers. 2020 Open Access This article is distributed under the terms of the Creative Commons Attribution 4.0 International License (https://creativecommons.org/licenses/by-nc/4.0/), which permits unrestricted use, distribution, and non-commercial reproduction in any medium, provided you give appropriate credit to the original author(s) and the source, provide a link to the Creative Commons license, and indicate if changes were made. The Creative Commons Public Domain Dedication waiver (http://creativecommons.org/publicdomain/zero/1.0/) applies to the data made available in this article, unless otherwise stated. 
of PHPT. The patient now endorsed gastroesophageal reflux, nephrolithiasis, nocturia, urinary frequency, constipation, weight gain, and fatigue. He denied any bone pain, bone fractures, osteoporosis, osteopenia, changes in alertness, or depression.

This patient's chronic medical conditions included hypertension, gastroesophageal disease, and vitamin D deficiency. He denied any history of neck surgeries and denied a family history of thyroid carcinoma, thyroid disease, parathyroid disease, or MEN syndromes. On physical exam, his thyroid was normal in size without palpable nodules or tenderness. There was no associated cervical or supraclavicular lymphadenopathy.

The patient underwent a thyroid ultrasound at LUMC. The right thyroid lobe measured $5.8 \times 1.8 \times 2.2 \mathrm{~cm}$ and the left thyroid lobe measured $5.0 \times 2.6 \times 1.8 \mathrm{~cm}$. No nodules within the thyroid were identified. A prominent left level III possible lymph node was identified measuring $1.4 \times 0.6 \mathrm{~cm}$ with a small fatty hilum. Parathyroid nuclear scan was recommended per guidelines. ${ }^{10}$ Sestamibi with SPECT/CT imaging demonstrated a focal area of uptake in the left neck at the level II-III region just deep to the sternocleidomastoid muscle, which was suspicious for a parathyroid adenoma in the setting of the patient's clinical history and biochemical workup.

The patient consented to a left upper neck exploration and subtotal parathyroidectomy with excision of an ectopic left UPA, which was performed at LUMC. A left oblique upper neck incision was made just below the level of the left submandibular gland. An ectopic, enlarged parathyroid gland was found just lateral to the internal jugular vein, as visualized on preoperative imaging. The gland was carefully dissected out and removed to be sent to pathology, which reported a hypercellular parathyroid gland weighing $0.74 \mathrm{~g}$ (Fig. 1). Intraoperative PTH levels decreased from 239 to $48 \mathrm{pg} / \mathrm{mL} 10$ minutes after excision of the left ectopic UPA.

The patient was monitored overnight in the observation unit, per surgeon protocol, and had an uncomplicated postoperative recovery. He returned to the clinic the following week for an initial postoperative visit. He denied numbness or tingling in his fingertips, neck discomfort, dysphagia, voice changes, and reported no complaints regarding health overall. The left upper neck incision site was healing well without swelling, erythema, or drainage. His calcium level was $10.0 \mathrm{mg} / \mathrm{dL}$ and phosphorus level was $3.7 \mathrm{mg} / \mathrm{dL}$.
The patient was discharged from our care with counseling on incision care and to return to clinic as needed.

\section{Conclusion}

Although UPAs are rare, most endocrine surgeons encounter them in their practice. It is thus important to have a thorough understanding of the necessary workup and options in the surgical approach to achieve the best outcomes. Deliberate preoperative imaging and planning made it possible for the patient in this case report to undergo curative minimally invasive parathyroidectomy with a resolution of symptoms and normalization of calcium and PTH levels.

\section{Clinical Significance}

Previous reports of UPAs fall within two categories: ectopic glands found in first-time explorations and those found in subsequent explorations. Undescended parathyroid adenomas have a higher prevalence in reoperative cases, generally because they cause failed primary surgeries. The case presented in this case report is unique because it is a primary case on a previously unoperated neckrates of UPAs in primary cases are $<1 \% .^{11}$ Much of the published data on UPAs focuses on recurrent or persistent cases, rather than primary explorations such as this case report., ${ }^{1,11}$ Table 1 summarizes previous studies that have reported UPAs in reoperative cases.

Ectopic parathyroid adenomas are clinically relevant because they can lead to failed neck explorations and cause persistent PHPT, which occurs in about $5-10 \%$ of initial parathyroidectomies. Repeat cases have increased complication rates due to the presence of scar tissue that violates normal planes and makes reoperation difficult. ${ }^{6}$ Undescended parathyroid adenomas are particularly challenging, because they are less accurately identified on preoperative imaging. ${ }^{1}$ This can lead to longer operative time and increased risk of injury to surrounding structures during an extensive dissection. ${ }^{8}$ In this case report, strategic preoperative imaging was used to excise the ectopic gland in a time-efficient manner and with minimal dissection.

Imaging before surgery is imperative in guiding the operative management of biochemically diagnosed PHPT, especially while localizing ectopic parathyroids. Guidelines recommend that patients first undergo laboratory testing of calcium and PTH levels,
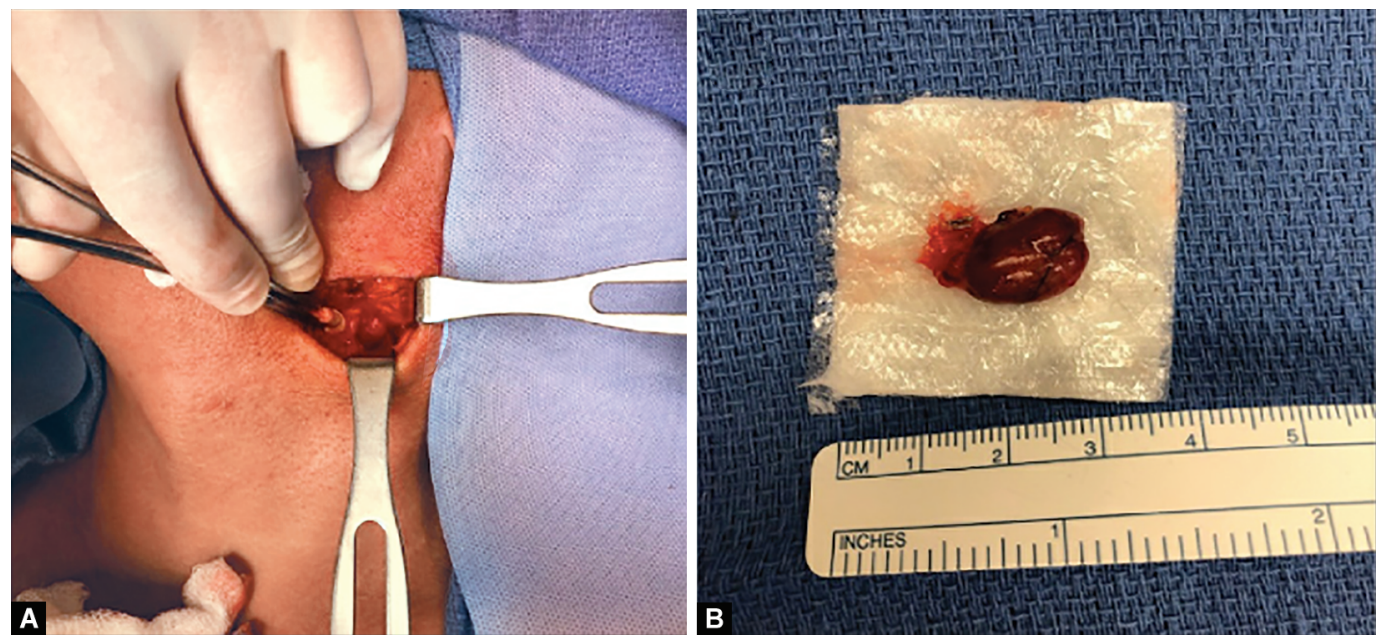

Figs $1 \mathrm{~A}$ and B: (A) Exposure of undescended adenoma through transverse left upper neck incision; (B) Surgical specimen: undescended parathyroid adenoma measuring approximately $1.5 \mathrm{~cm} \times 1 \mathrm{~cm}$ 
then cervical ultrasonography. The second most recommended imaging is technetium Tc $99 \mathrm{~m}$ sestamibi. ${ }^{10}$ However, sestamibi scans have low sensitivity and report false-negative results in $11-33 \%$ of cases with a single parathyroid adenoma. ${ }^{12}$ In a study by Lee et al., patients with UPAs who underwent a second imaging test in addition to sestamibi routinely had successful minimally invasive parathyroidectomies, compared to $17 \%$ success for those patients that only had sestamibi. ${ }^{1}$ The patient presented in this case report underwent sestamibi imaging with SPECT/CT. Figure 2 demonstrates the patient's imaging that showed an ectopic parathyroid adenoma in levels 2-3 of the left neck.

Several other diagnostic studies can evaluate ectopic parathyroid glands. Mahajan and Schoder described a case in which 3-dimensional imaging SPECT/CT accurately localized a previously missed UPA that had been mistaken for a submandibular gland. ${ }^{13}$ Recommended intraoperative adjuncts include internal jugular venous sampling to find PTH gradients and gamma probe use. ${ }^{1}$ A study by Axelrod et al. reviewed non-diagnostic preoperative imaging after removal of a UPA and noted asymmetry in the submandibular glands, hinting on the laterality of the ectopic gland previously unidentified before surgery. ${ }^{12}$ While the UPA in this case report was not mistaken for a submandibular gland, it was initially thought to be a lymph node due to its level in the neck. Further CT-guided imaging made it suspicious for a parathyroid adenoma.

The gold standard for treatment of PHPT has been bilateral neck exploration to surgically excise parathyroid adenomas, which has a cure rate of 90-97\% when performed by experienced surgeons. ${ }^{14}$ Recent literature has argued for routine preoperative localization to dictate minimally invasive parathyroid surgery, using smaller incisions and unilateral neck exploration to increase

Table 1: Prevalence of undescended parathyroid adenoma (UPA) in reoperative cases $9,12,13$

\begin{tabular}{llll}
\hline Study & Year published & $\begin{array}{l}\text { \# of UPA/Total \# } \\
\text { of patients }\end{array}$ & $\begin{array}{l}\text { Prevalence of } \\
\text { UPA (\%) }\end{array}$ \\
\hline Fraker et al. & 1990 & $9 / 145$ & 6.2 \\
Billingsley et al. & 1994 & $17 / 255$ & 6.7 \\
Shen et al. & 1996 & $9 / 102$ & 8.8 \\
Jaskowiak et al. & 1996 & $18 / 222$ & 8.1 \\
\hline
\end{tabular}
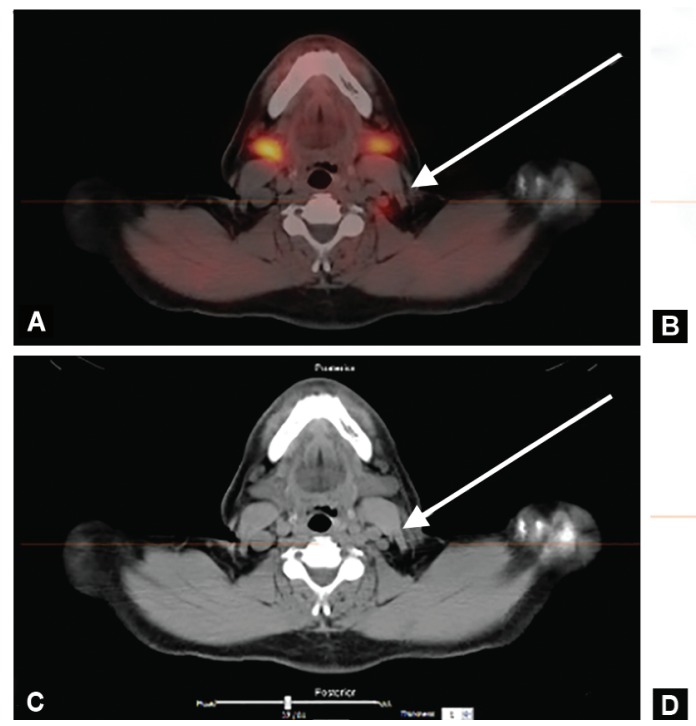

cost-effectiveness and decrease patient morbidity. ${ }^{15}$ This also allows surgeons to deviate from the textbook approach and make incisions directly over the ectopic adenomas. For example, previous studies have reported incisions directly over the submandibular triangle, at the level of the hyoid bone for a retropharyngeal UPA, or over the carotid sheath for an intravagal adenoma. ${ }^{1}$

The patient in this case report only required cervical ultrasound and sestamibi with CT for the surgeons to directly access the UPA with a well-informed, targeted incision that deviated from the traditional midline cervical approach. Intraoperative adjuncts such as gamma probe were not necessary, although a $>50 \%$ decline in intraoperative PTH levels was key to determining successful excision of a hypercellular parathyroid adenoma. This facilitated a unilateral approach and ruled out the need for bilateral exploration. Strategic preoperative imaging combined with surgeon experience allowed the patient to undergo a time-efficient, curative operation followed by an uncomplicated postoperative recovery.

\section{References}

1. Lee JC, Mazeh H, Serpell J, et al. Adenomas of cervical maldescended parathyroid glands: pearls and pitfalls. ANZ J Surg 2015;85(12): 957-961. DOI: 10.1111/ans.12017.

2. Phitayakorn R, McHenry CR. Incidence and location of ectopic abnormal parathyroid glands. Am J Surg 2006;191(3):418-423. DOI: 10.1016/j.amjsurg.2005.10.049. Available from: https://www. americanjournalofsurgery.com/article/S0002-9610(05)00929-3/ fulltext\#.

3. Roy M, Mazeh $\mathrm{H}$, Chen $\mathrm{H}$, et al. Incidence and localization of ectopic parathyroid adenomas in previously unexplored patients. World J Surg 2013;37(1):102-106. DOI: 10.1007/s00268-012-1773-z.

4. Bliss RD, Gauger PG, Delbridge LW. Surgeon's approach to the thyroid gland: surgical anatomy and the importance of technique. World J Surg 2000;24(8):891-897. DOI: 10.1007/s002680010173.

5. Theurer S, Siebolts U, Lorenz K, et al. Ectopic tissue of the thyroid gland and the parathyroid glands [Internet]. Pathologe 2018;39(5):379-389. DOI: 10.1007/s00292-018-0467-1.

6. Chan TJ, Libutti SK, McCart JA, et al. Persistent primary hyperparathyroidism caused by adenomas identified in pharyngeal or adjacent structures. World J Surg 2003;27(6):675-679. DOI: 10.1007/ s00268-003-6812-3.

7. Nguyen J, Lau O, Abemayor E, et al. Undescended parathyroid adenoma arising within the hypoglossal nerve. Arch Otolaryn Head Neck Surg 2011;137(7):709-711. DOI: 10.1001/archoto.2011.90.
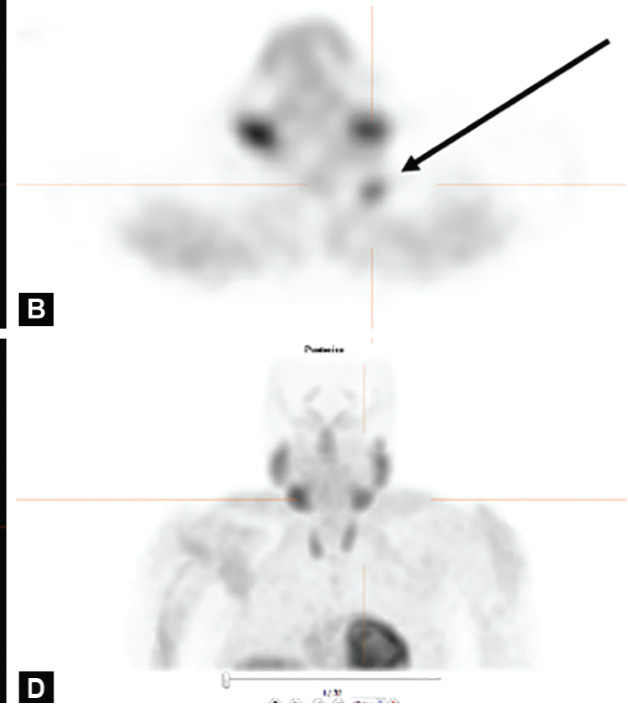

Figs 2A to D: (A) SPECT/CT image of undescended adenoma (axial view); (B) Sestamibi scan of undescended adenoma (axial view); (C) CT image of undescended adenoma (axial view); (D) Sestamibi scan of undescended adenoma (coronal view) 
8. Kanack MD, Maawy AA, Oh DK, et al. Undescended parathyroid adenoma. BMJ Case Rep 2015;2015(mar03 1):2014-2016. DOI: 10.1136/ bcr-2014-208277.

9. Fraker DL, Doppman JL, Shawker TH, et al. Undescended parathyroid adenoma: an important etiology for failed operations for primary hyperparathyroidism. World J Surg 1990;14(3):342-348. DOI: 10.1007/ BF01658522.

10. Wilhelm SM, Wang TS, Ruan DT, et al. The American Association of Endocrine Surgeons guidelines for definitive management of primary hyperparathyroidism. JAMA Surg 2016;151(10):959-968. DOI: 10.1001/ jamasurg.2016.2310.

11. Rioja P, Mateu G, Lorente-Poch L, et al. Undescended parathyroid adenomas as cause of persistent hyperparathyroidism. Gland Surg 2015;4(4):295-300. DOI: 10.3978/j.issn.2227-684X.2015.04.14.
12. Axelrod D, Sisson JC, Cho K, et al. Appearance of ectopic undescended inferior parathyroid adenomas on technetium Tc $99 \mathrm{~m}$ sestamibi scintigraphy: a lesson from reoperative parathyroidectomy. Arch Surg 2003;138(11):1214-1218. DOI: 10.1001/archsurg.138. 11.1214.

13. Mahajan S, Schoder H. Ectopic undescended parathyroid adenomaSPECT/CT avoids false-negative interpretation on $99 \mathrm{mTC}-\mathrm{MIBI}$ dualphase scintigraphy. Clin Nucl Med 2018;43(3):199-200. DOI: 10.1097/ RLU.0000000000001958.

14. Lee LS, Canter RJ, Fraker DL. Intraoperative jugular venous sampling aids detection of an undescended parathyroid adenoma. World $\mathrm{J}$ Surg 2006;30(4):620-623. DOI: 10.1007/s00268-005-0238-z.

15. Fahy BN, Bold RJ, Beckett $\mathrm{L}$, et al. Modern parathyroid surgery. Arch Surg 2002;137(8):917-923. DOI: 10.1001/archsurg.137.8.917. 